\title{
STELLAR WINDS IN A-TYPE SUPERGIANTS
}

\section{A. Talavera ${ }^{1 *} \quad$ A.I. Gomez de Castro ${ }^{2}$}

${ }^{1}$ ESA IUE Observatory, VILSPA P.O.Box 54065, 28080 Madrid, Spain
${ }^{2}$ Observatorio Astronomico Nacional. Alfonso XII, 3, 28014 Madrid, Spain

\section{INTRODUCTION}

The contribution of A supergiant stars to the return of mass and energy to the interstellar medium is not very important. Abbott (1982) analised a sample of early stars and concluded that $B$ and $A$ supergiants provided less than $8 \%$ of the mass input to the ISM by stellar winds. However, A-type supergiants are important in the framework of the stellar winds-mass loss phenomenology since they are located at the boundary between hot and cool stars, where radiative acceleration may not be sufficiently efficient to drive the wind.

We have studied the ultraviolet spectrum of all the $A$ type supergiants observed with IUE (Talavera and Gomez de Castro 1987, 1988). This work allowed us to classify them in two groups. The Group $I$ contains the less luminous A supergiants; these stars show indications of mass outflow only in the $\mathrm{Mg}$ II resonance lines where an absorption component of variable blueshift and intensity has been detected. The stars included in the Group II show strong evidence of wind and mass loss in the UV resonance lines of $\mathrm{Mg} \mathrm{II}$, C II, Si II and Fe II, and the terminal velocity of the wind derived from the $\mathrm{Mg}$ II lines is about $250 \mathrm{~km} / \mathrm{s}$; the most luminous A supergiants belong to this group.

There are also two important indicators of mass loss in the visible: a) emission : $\mathrm{H} \alpha$, $\mathrm{H} \beta$, He I $5876 \AA$, and b) dependence of the radial velocity on the excitation potencial of the lines and/or regular progression of the radial velocity along the Balmer series (Hutchings 1970, Wolf 1983).

The only systematic study of $\mathrm{H} \alpha$ in A supergiants was made by Rosendhal (1970). He found an evolution of the emission in the line with the absolute magnitude of the stars. $\mathrm{He}$ found emission in $\mathrm{H} \alpha$ fof magnitudes brighter than $\mathrm{Mv}=-6.8$.

In 1988 we started an observing programme whose goal is the detailed analysis of the envelope of the A type supergiants. As a first step we have obtained high resolution profiles of lines formed at different depths in the atmosphere of the stars. These data will allow us to model the wind and the structure of the outer layers of these stars.

We present here the first results of this programme.

\footnotetext{
*Affiliated to the Astrophysics Division, Space Science Department
} 


\section{OBSERVATIONS}

We have selected our stars from the compilation of Humphreys (1978). Since most of them belong to $\mathrm{OB}$ associations, we have a good estimate of the distance and therefore of the absolute magnitude. Part of the stars we studied with IUE are included in this first sample which is listed in Table 1.

The observations have been done with the Isaac Newton Telescope (INT) of the Observatorio del Roque de los Muchachos in the Canary Islands and with the $2.2 \mathrm{~m}$ telescope of the Calar Alto Observatory in September and October 1988. At the INT we used the Intermediate Dispersion Spectrograph (IDS) equipped with a GEC CCD and in Calar Alto we used the Coude Spectrograph with an RCA CCD.

The spectral intervals we have selected correspond to the following lines: $\mathrm{Ca}$ II $\mathrm{K}$ line $(3933 \AA), \mathrm{Mg}$ II $(4481 \AA), \mathrm{H} \beta(4861 \AA), \mathrm{Na}$ I and $\mathrm{He} \mathrm{I}(5890 \AA)$ and $\mathrm{H} \alpha(6563 \AA)$. We have observed all these lines in most of the stars of the sample with a resolving power around 20000 .

Table 1. Ho Observations of A-type supergiants

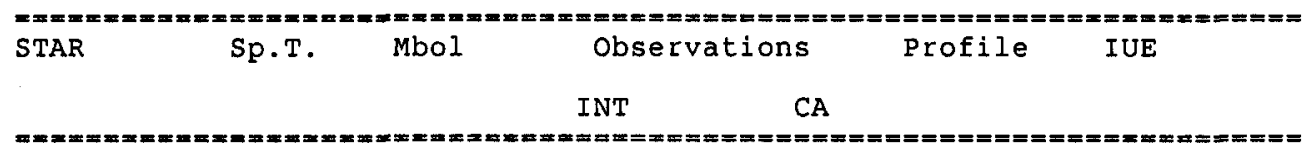

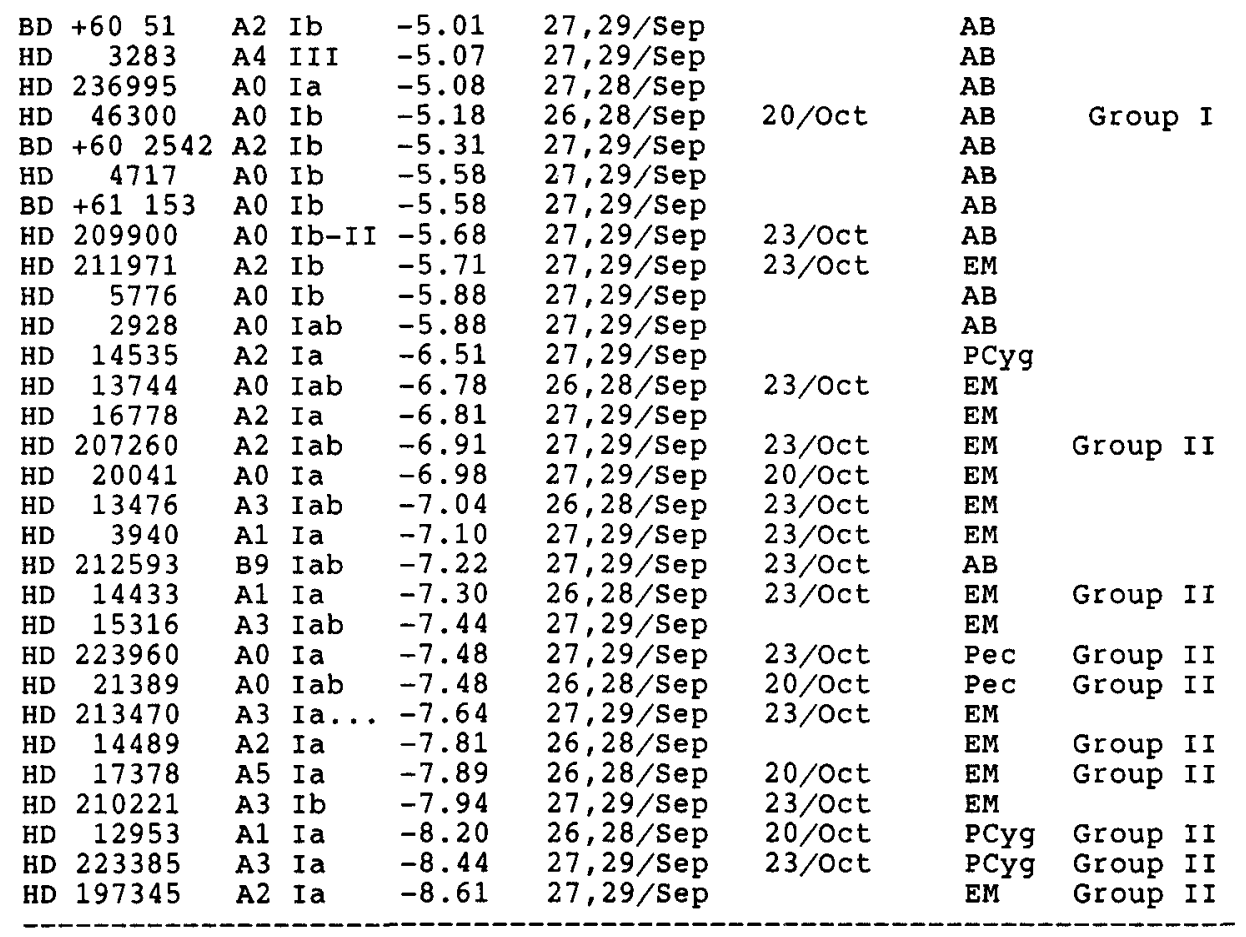

Mbol and spec. Type from Humphreys (1978); INTrIsaac Newton Telescope; $\mathrm{CA}=2.2 \mathrm{~m}$ Calar Alto; all the observing dates are 1988 . 


\section{PRELIMINARY RESULTS: H $\alpha$}

\subsection{Data reduction}

The spectra have been extracted from the CCD frames after dark noise correction and flatfielding. The one dimensional spectra so obtained have been rebinned into a wavelength scale by using a polinomial fit to the positions provided by the thorium-argon lines of the comparison lamp.

The resolution measured in the comparison spectra is $0.25 \AA(11 \mathrm{~km} / \mathrm{s}$ at $\mathrm{H} \alpha)$ and 0.40 $\AA(18 \mathrm{~km} / \mathrm{s})$ in Calar Alto and the INT respectively. Both set of data have a signal to noise ratio ranging between 100 and 200.

All the spectra have been divided by a linear continuum placed by eye.

The data have been processed using the IHAP and MIDAS systems.

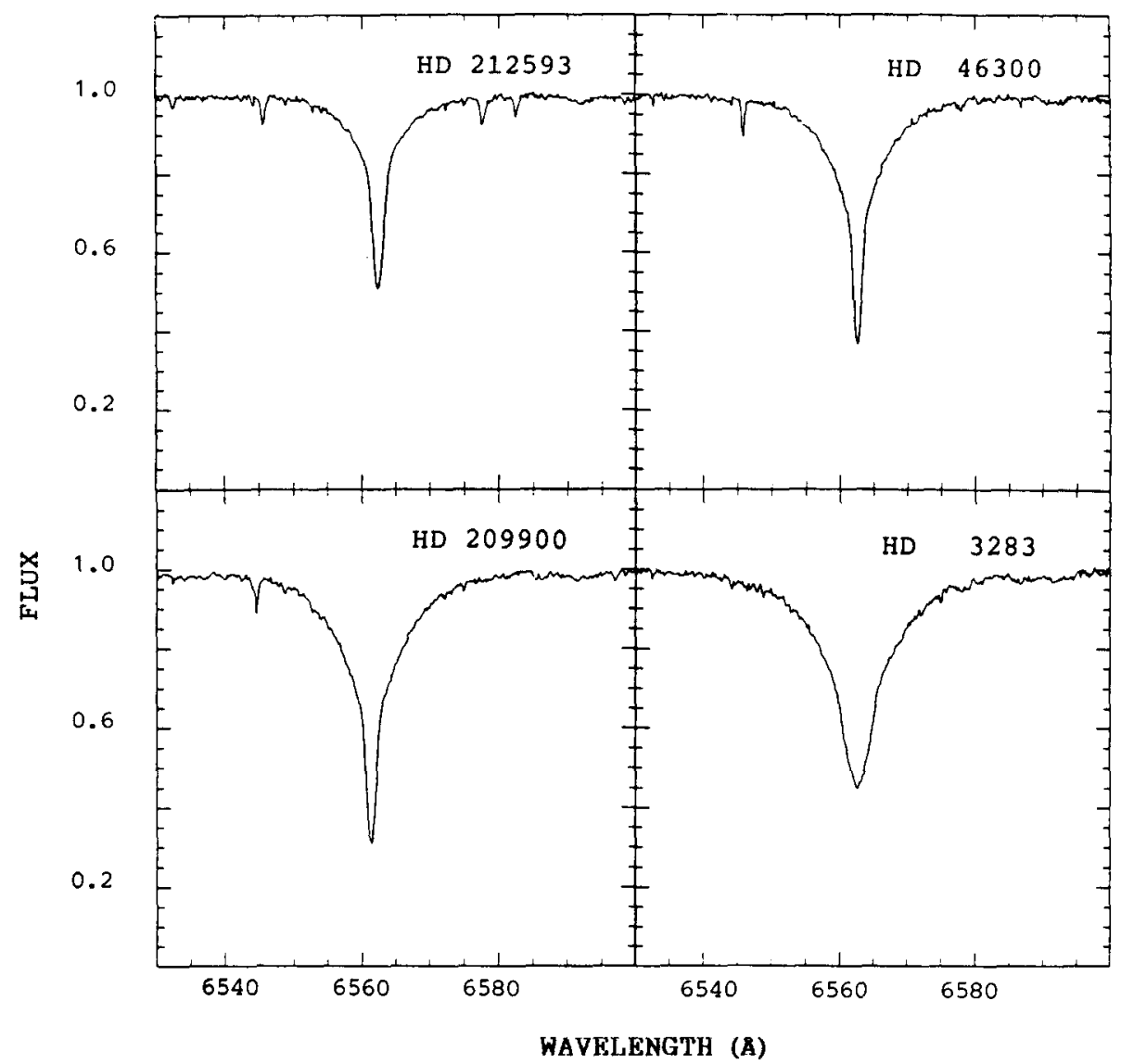

Fig.1. Ha profiles in some of the less luminous A supergiants. 


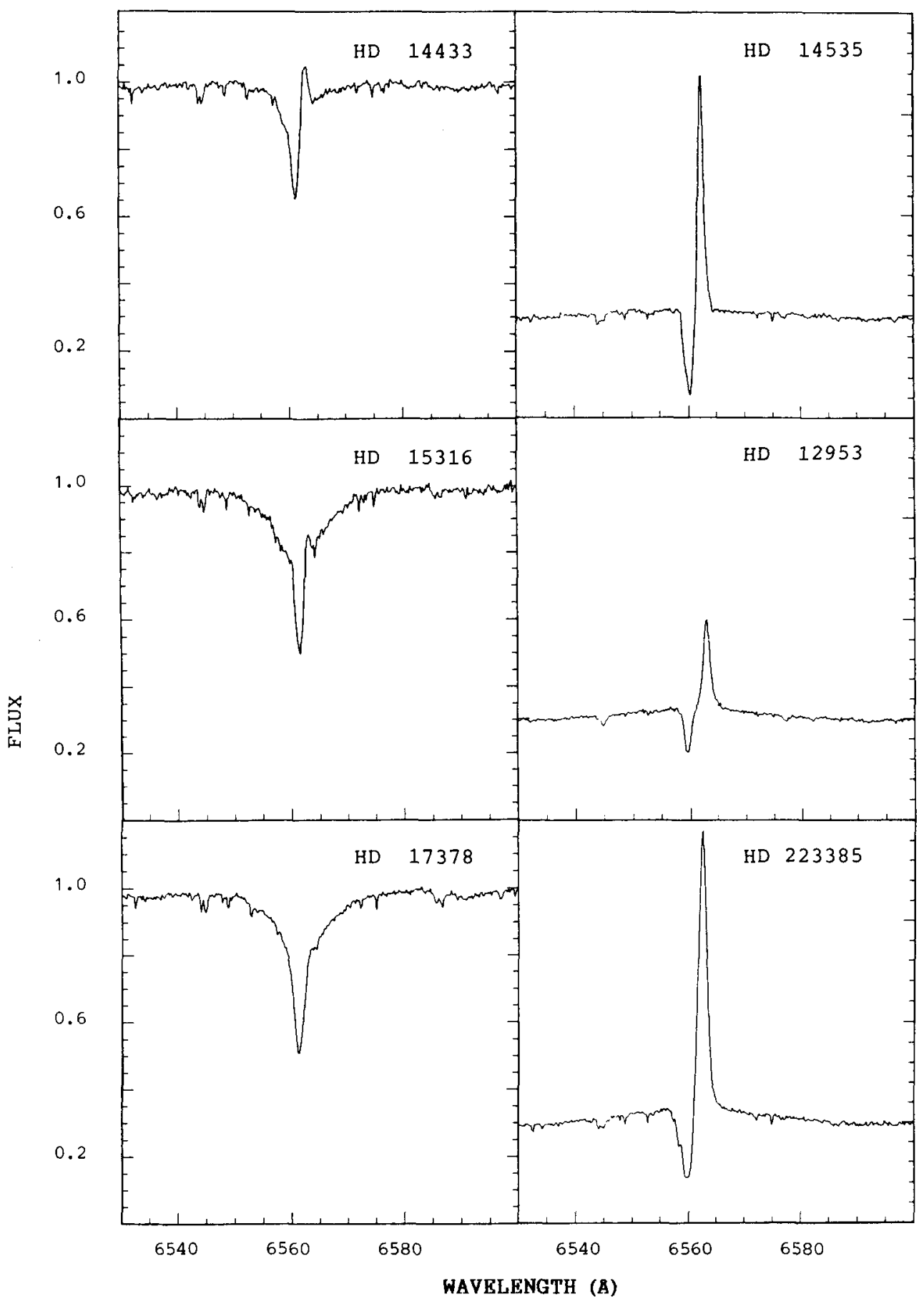

Fig.2. Evolution of the $\mathrm{Ha}$ line in luminous A supergiants. 


\subsection{Observed profiles}

The $H \alpha$ profiles in A-type supergiants present very different shapes going from pure symmetric absorption to emission. The observed profile type is given for each star in Table 1. From that table it can be seen that the profiles evolve according to the absolute magnitude of the stars as it was noticed by Rosendhal (1970). This trend is ilustrated in Figs. 1 and 2. The less luminous stars show symmetric absorption profiles; then when luminosity increases an asymmetry starts developing in the core of the absorption which becomes a P Cyg type III profile for the brightest stars. In two "peculiar" stars the observed profile is in pure emission.

The less luminous stars do not show variability in the profiles, which have been observed for all the stars at least twice, at different epochs (see Table 1). However we have observed variations in some of the stars showing asymmetric-emission profiles. Some of these variations are very strong. They will be studied in future work.

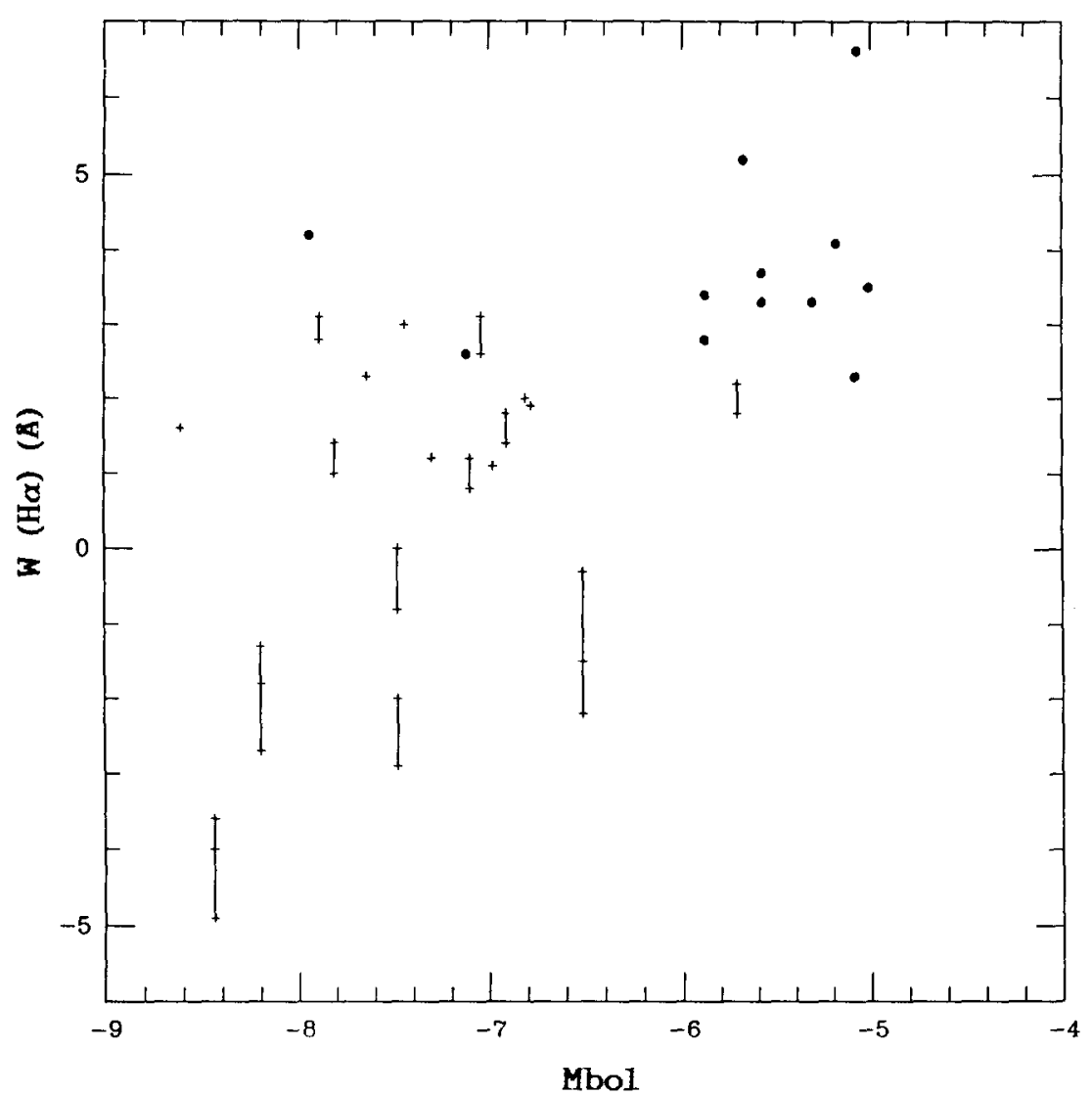

Fig.3. Equivalent width of $\mathrm{H} \alpha$ versus bolometric magnitude. Symmetric absorption lines are represented with dots and lines showing emission with crosses. Vertical lines indicate observed variation. 


\subsection{Equivalent widths}

We have measured the equivalent width of the observed $\mathrm{H} \alpha$ lines. The results are ilustrated in Fig. 3, where we have represented $\mathrm{W}(\mathrm{H} \alpha)$ against $\mathrm{Mbol}$. The reported values correspond to absorption below the continuum minus emission above the continuum. In that figure we have indicated the variations observed in the equivalent width by vertical lines.

Although we cannot say there is a clear correlation $\mathrm{W}(\mathrm{H} \alpha)$ - Mbol, it can be seen from Fig. 3 that there is a trend of increasing equivalent width with increasing magnitude. In other words the absorption in $\mathrm{H} \alpha$ is cancelled by emission in the core of the line when we go to more luminous stars. We have measured also the emission peak intensity for the stars showing that feature and we have obtained also the same trend.

From our sample of stars we can say that emission in the core of $\mathrm{H} \alpha$ is observed for stars brighter than $\mathrm{Mv}=\mathbf{- 5 . 5}$.

\subsection{Comparison with the U. V.}

The sample of stars we have observed is larger than the one on which we based our studies with IUE. The first result of our new observations in $\mathrm{H} \alpha$ is the confirmaton of the division between Groups I and II established from IUE data. The less luminous A supergiants which showed weak signs of stellar wind in the U.V., those included in group I, have Ha in absorption, while the stars of group II, the most luminous ones, present emission signs in the $\mathrm{H} \alpha$ profiles.

\section{CONCLUSIONS}

We have presented some very preliminary results of a large programme intended to study the winds of A-type supergiants. Our data confirm and extend to a larger sample the results pointed out by Rosendhal (1970) and provide further evidence for the conclusions derived from our work in the U.V.

The project is in progress and we are continuing the analysis of the lines mentioned in Section 2 . In the second part of our project we shall try the modelling of the envelope-wind complex in A-type supergiants.

\section{REFERENCES}

Abbott, D.C. 1982, Astrophys.J., 263, 723

Humphreys, R.M. 1978, Astrophys.J.Suppl., 38, 309

Hutchings, J.B. 1970, Mon.Not.Roy.Astr.Soc., 147, 161

Rosendhal, J.D. 1970, Astrophys.J., 186, 909

Talavera, A., and Gomez de Castro, A.I.1987, Astron.Astrophys., 181, 300

Talavera, A., and Gomez de Castro, A.I.1988, in Proceedings of The 10th European Regional Astronomy Meeting 'of the IAU, ed. P. Harmanec, Vol. 5, p.207

Wolf, S.C. 1983, in The A-type stars: problems and perspectives, NASA SP-463, p.118 